\title{
Isolation and molecular identification of Leishmania chagasi from a bat (Carollia perspicillata) in northeastern Venezuela
}

\author{
Hector De Lima/ ${ }^{+}$, Noris Rodríguez, Miguel A Barrios, Ángela Ávila, Israel Cañizales ${ }^{1}$, Saúl Gutiérrez ${ }^{1}$ \\ Instituto de Biomedicina, Universidad Central de Venezuela, Apartado 4043, 1010A Caracas, Venezuela ${ }^{1}$ Geoclean C.A., \\ Caracas, Venezuela
}

This report describes the isolation of a Leishmania chagasi strain from a bat (Carollia perspicillata), and its identification using biological methods and molecular characterization. The parasites were isolated in an artificial culture medium from a blood sample extracted from a bat heart. The isolate was then inoculated into the footpads of Balb/c mice, which subsequently developed a typical nodular leishmanial lesion; the parasites were confirmed as Leishmania by smear and histopathology. Molecular characterization of the parasites was performed by polymerase chain reaction with species-specific primers, $k D N A$ restriction pattern following Hae III endonuclease digestion and dot blot hybridization using a kDNA probe. This report demonstrates that bats can be hosts for L. chagasi species and suggests the need for studies to determine whether they may be involved in foci of visceral leishmaniasis.

Key words: Leishmania chagasi - bats - molecular biology - reservoir - Venezuela

Leishmania present a complex life cycle that involves vectors, reservoirs and susceptible hosts that vary according to the ecological conditions where they develop. A great variety of mammals, including more than 100 species, both domestic and wild, have been suggested as potential reservoirs for Leishmania (WHO, 1990). However, it has not been possible to demonstrate the participation of Chiroptera in the Leishmania life cycle, even though they are a relatively large group of mammals present in practically all the ecosystems, including urban and peri-urban environments. There are limited studies investigating the role of bats in the Leishmania life cycle. In the Old World, indirect evidence suggests bats as potential Leishmania reservoirs. Mutinga (1975) demonstrated Leishman-Donovan bodies in 3/104 bats in Kenya, and Morsy et al. (1987) detected anti-Leishmania antibodies in seven bats in Egypt. The frequent presence of phlebotomine sandflies in caves and crevices inhabited by bats suggests that bats may provide an alternative blood support in such niche environments (KillickKendrick et al. 1977, Lampo et al. 2000). In Venezuela, Lampo et al. (2000) used bats undergoing feeding trials to demonstrate four species of bats as a blood source for Lutzomyia longipalpis, the main vector of the Leishmania species that causes American visceral leishmaniasis (VL). Rotureau et al. (2006), in a study performed in 216 bats representing 29 species from cutaneous leishmaniasis (CL) foci in French Guiana, found no evidence of Leishmania after a PCR analysis with genus-specific primers and culture of animal samples.

Financial support: Geoclean and Sincrudos de Oriente (SINCOR)

+ Corresponding author: delimah@gmail.com

Received: 29 March 2008

Accepted: 20 June 2008
In this report, we isolated Leishmania chagasi from a Carollia perspicillata bat and then identified it using biological characteristics and molecular techniques. The study was performed in and around the SINCOR Biological station facility within the Orinoco Oil Belt. This region is located in the Monagas municipality in the South of the state of Anzoategui, Venezuela (8 $8^{\circ} 18^{\prime} 09^{\prime \prime}-$ $8^{\circ} 21^{\prime} 48^{\prime \prime} \mathrm{N}$ and $\left.64^{\circ} 56^{\prime} 51^{\prime \prime}-65^{\circ} 01^{\prime \prime} 35^{\prime \prime} \mathrm{W}\right), 130-164 \mathrm{~m}$ a.s.1., located predominantly in the tropical dry forest zone. Annual temperatures range $22-29^{\circ} \mathrm{C}$ and the annual precipitation is approximately $1,000 \mathrm{~mm}$.

Bats were captured during one week in August 2006 using two methods, depending on the time of day. Manual direct capture was used during noon hours prior to location of diurnal roosts, and mist nets were used for capture during the night. Taxonomical identification was performed according to Emmons' field guide (1997). The skin of the animals was examined externally for the presence of suspicious lesions. Blood samples $(1 \mathrm{ml})$ were taken from the heart of each animal under ether anesthesia, and the samples were used for fresh microscopic examination, Giemsa stained smears and culture in blood agar base medium (Difco) containing 10\% defibrinated rabbit blood and $200 \mathrm{U}$ penicillin. From positive cultures, $10^{6}$ parasites were inoculated subcutaneously into the ventral pad of the left hind foot of Balb/c mice of the same sex, weight and age $(n=5)$. Molecular characterization of the parasites consisted of (i) PCR using AJS-3 and DBY species-specific primers that are specific for the Leishmania donovani complex (Lambson et al. 1999, 2000); (ii) dot-blot hybridization using total kDNA probes designed based on the international reference strain L. chagasi; and (iii) kDNA restriction patterns following Hae III endonuclease digestion, as previously described by Rodriguez et al. (2005).

A total of 11 bats were captured, all belonging to one family (Phyllostomidae) but distributed in three genera: Carollia (8), Phylloderma (2) and Rhynophylla (1). None of the bats showed lesions upon external physical exami- 
nation. All fresh blood microscopic examinations and Giemsa stained blood smears were negative for hemoparasites. A blood culture was positive for Leishmania promastigotes in one animal identified as "M9", a female Carollia perspicillata (MCAR/VE/06/M9). Mice inoculated with promastigotes from the M9 isolate developed a nodular lesion in the inoculated footpad. The lesions were progressive, reaching $5 \mathrm{~mm}$ (average size) after one month and the parasites were successfully maintained in mice. The histopathological study revealed a macrophage-rich granuloma laden with amastigotes (Fig. 1).

Gel electrophoresis of the PCR amplicon from the M9 isolate showed the expected diagnostic band of approximately 800 bp (Fig. 2A, line 4), which was also observed in the PCR products from samples of the international reference strains $L$. infantum and L. chagasi (Fig. 2A, lines 2,3), although the M9 product also contained additional bands. No product was seen by PCR using primers for Leishmania and the Viannia subgenus (data not shown). Dot blot hybridization of the PCR product with total kDNA from $L$. chagasi showed a strong hybridization signal with M9 (Fig. 2B, sample 4). Additionally, kDNA analysis with the Hae III restriction enzyme showed strong homology between the M9 restriction pattern and L. chagasi (Fig. 3, lines 4, 3 respectively), which shared products of approximately $850 \mathrm{bp}$, bands between 650 and $500 \mathrm{bp}$ and one small band of $300 \mathrm{bp}$.

In this work we report the casual finding of a bat infected with $L$. chagasi parasites, which suggests a possible role of bats in the leishmaniasis epidemiological cycle. Until now, indirect inconclusive evidence has associated bats with the Leishmania life cycle, although the parasites have never been directly identified in bats (Mutinga 1975, Morsy et al. 1987). This is likely due to outdated techniques and the paucity of studies investigating Leishmania in bats. In contrast, Chiroptera have been found infected with a variety of species of trypanosomes, including Trypanosoma cruzi, the causative agent of Chagas disease (Barnabe et al. 2003). The Leishmania

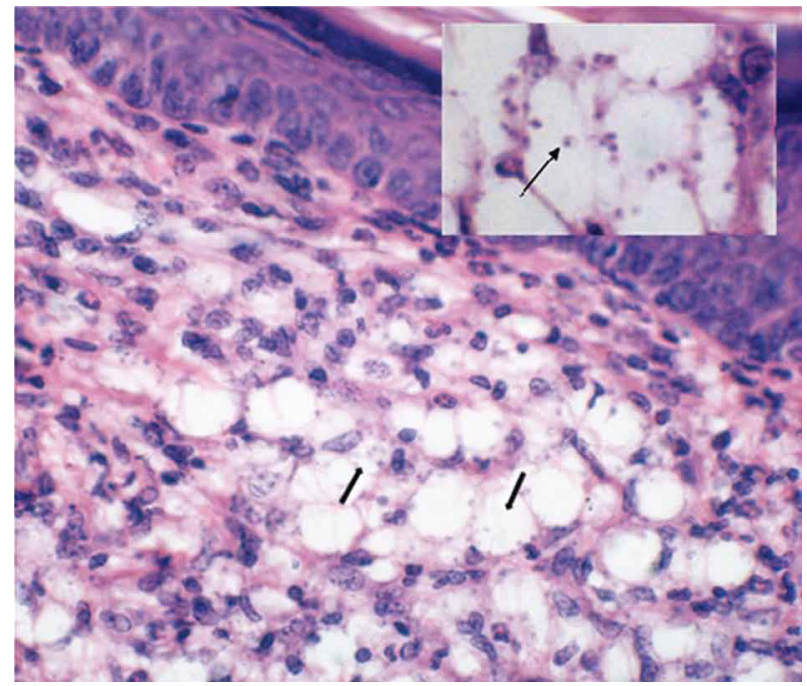

Fig. 1: histological section of nodular lesion in a mouse footpad showing the diffuse dermal infiltration of vacuolated macrophages (arrows) with abundant amastigotes, 400X. In the detail: amastigotes, 630X.

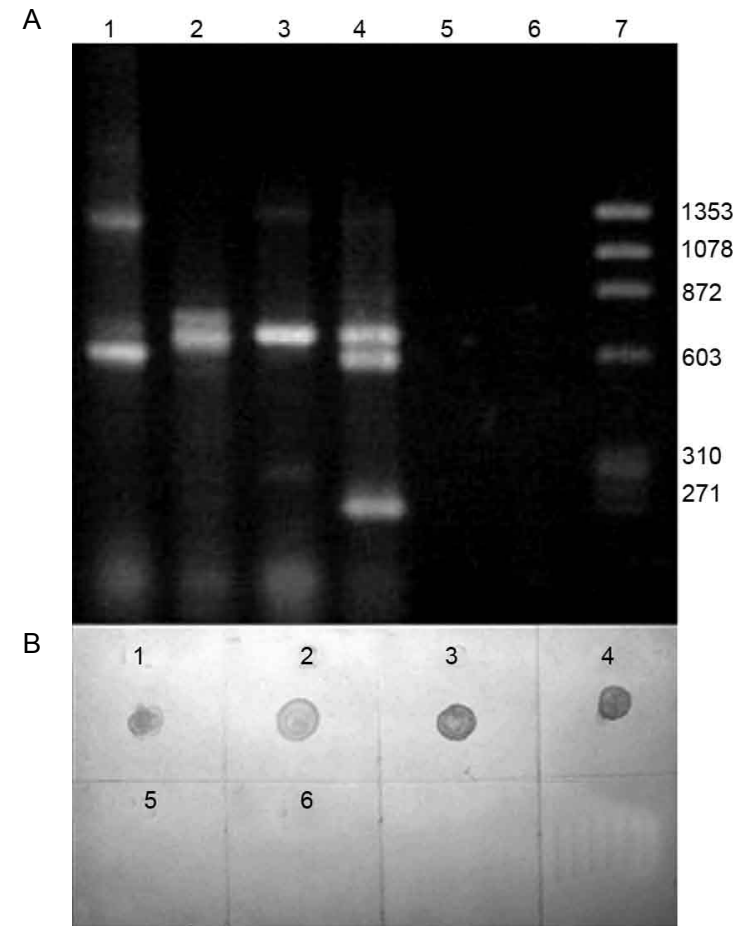

Fig. 2: A: gel electrophoresis in 1\% agarose of the PCR products obtained after 35 amplification cycles with AJS-3 and DBY Leishmania donovani species specific primers. B: dot blot hybridization of PCR product hybridized with kDNA from Leishmania chagasi. Lanes and squares - 1: L. donovani (MHOM/IN/80/DD8); 2: Leishmania infantum (MHOM/TN/80/IPT-1); 3: L. chagasi (MHOM/Br/74/PP75); 4: bat isolate (MCAR/VE/06/M9); 5: Leishmania amazonensis (PH-8); 6: Leishmania braziliensis (M2903); 7: molecular weight markers ( $\Phi$ X174 DNA, Gibco BRL).

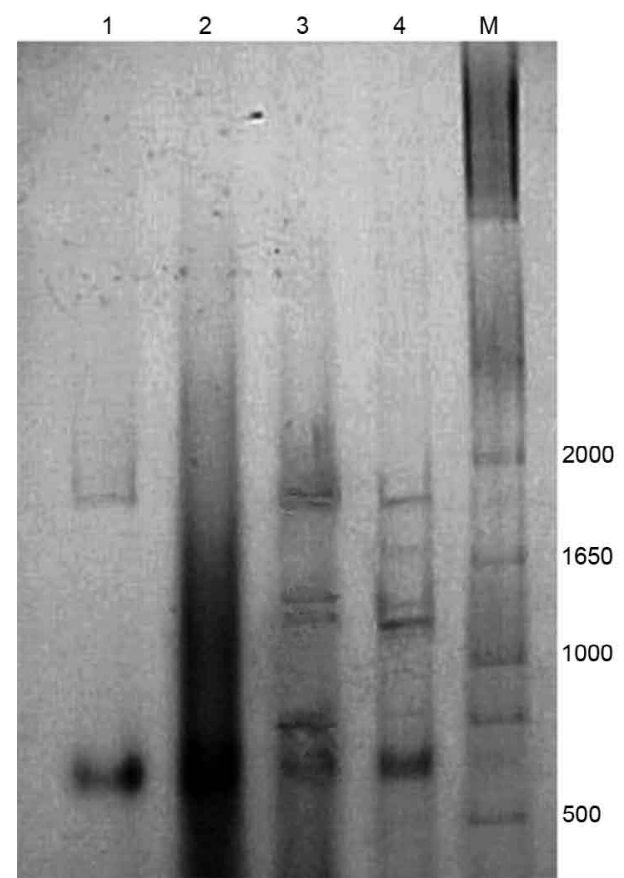

Fig. 3: polyacrylamide gel electrophoresis of total kDNA digested with Hae III restriction enzyme. Lane - 1: Leishmania donovani (MOHM/ IN/80/DD8); 2: Leishmania infantum (MHOM/TN/80/IPT-1); 3: Leishmania chagasi (MHOM/Br/74/PP75); 4: bat isolate (MCAR/ VE/06/M9); M: molecular weight markers (1Kb Plus, Gibco BRL). 
infected bat was captured in an unpopulated area of Monagas in the state of Anzoategui. This may be significant considering that, of the 423 cases of VL reported in Venezuela in the last 10 years (1998-2007), $76(17.96 \%)$ were diagnosed in this state, with no cases being reported in Monagas. Additionally, according to the Venezuelan Ministry of Health, 1146 cases of CL were reported in Anzoategui during the same period, with sporadic cases in Monagas.

This report documents a specific case where a bat harbored a $L$. chagasi species, possibly due to the coexistence of Lu. longipalpis and bats in the same resting places (Killick-Kendrick et al. 1977, Lampo et al. 2000). Further studies of VL foci are needed to clarify the role of bats as possible reservoirs in the epidemiological chain of this disease.

\section{ACKNOWLEDGMENTS}

To Raimundo Rodríguez and Rodrigo Rueda for his technical assistance, Ms. Maria Eugenia Gallinoto and Howard Takiff for the English correction of the manuscript.

\section{REFERENCES}

Barnabe C, Brisse S, Tibayrenc M 2003. Phylogenetic diversity of bat trypanosomes of subgenus Schizotrypanum based on multilocus enzyme electrophoresis, random amplified polymorphic DNA, and cytochrome b nucleotide sequence analyses. Infect Genet Evol 2: 201-208.

Emmons LH 1997. Neotropical Rainforest Mammals: A field Guide, 2nd ed., The Universirty Chicago Press, Chicago, 298 pp.
Killick-Kendrick R, Leaney AJ, Ready PD 1977. The establishment, maintenance and productivity of a laboratory colony of Lutzomyia longipalpis (Diptera: Psychodidae). J Med Entomol 13: 429-440.

Lambson M, Smyth A, Barker D 1999. Sequence homology within a minicircle class of the Leishmania donovani complex. Mol Biochem Parasitol 101: 229-232.

Lambson M, Smyth A, Barker D 2000. Leishmania donovani: Development and characterization of a kinetoplast DNA probe and its use in the detection of parasites. Exp Parasitol 94: 15-22.

Lampo M, Feliciangeli MD, Marquez LM, Bastidas C, Lau P 2000. A possible role of bats as a blood source for the Leishmania vector Lutzomyia longipalpis (Diptera: Psychodidae). Am J Trop Med Hyg 62: 718-719.

Morsy TA, Salama MMI, Abdel Hamid MY 1987. Detection of Leishmania antibodies in bats. J Egypt Soc Parasitol 17: 797-798.

Mutinga MJ 1975. The animal reservoir of cutaneous leishmaniasis on Mount Elgon, Kenya. East Afr Med J 52: 142-151.

Rodriguez NM, De Guglielmo Z, Barrios MA, Barrios RM, Zerpa O, Feliciangeli MD 2005. Genetic homogeneity within Leishmania (L.) infantum isolated from human and dogs: the relationship with the sandfly fauna distribution in endemic areas of Nueva Esparta State, Venezuela. Parasitology 130: 611-619.

Rotureau B, Catzeflis F, Carme B 2006. Short Report: Absence of Leishmania in Guianan Bats. Am J Trop Med Hyg 74: 318-321.

WHO - World Health Organization 1990. Control of the leishmaniases, Report of a WHO Expert Committee, Technical Report Series 793, Geneva, p. 9-32. 